\title{
Principles of redox control in photosynthesis gene expression
}

\author{
Thomas Pfannschmidta , John F. Allen ${ }^{\mathrm{b}}$ and Ralf Oelmüller ${ }^{\mathrm{a}, *}$ \\ ${ }^{a}$ Institute of General Botany, Department of Plant Physiology, University of Jena, Dornburger Str. 159, D-07743 Jena, Germany \\ ${ }^{\mathrm{b}}$ Plant Biochemistry, Lund University, Box 117, SE-22100 Lund, Sweden \\ *Corresponding author, e-mail: b7oera@hotmail.com
}

Received 28 August 2000; revised 12 December 2000

Light is one of the most important environmental factors influencing gene expression in photosynthetic organisms. In particular, genes for components of the photosynthetic machinery show light-dependent expression. In recent years, it has become clear that photosynthesis itself contributes important signals to this light control of gene expression by means of changes in the reduction/oxidation (redox) state of signalling molecules. Such changes in redox state are induced by changes in quality and quantity of the incident light. Redox signalling mechanisms therefore provide photosynthesis with the possibility of acclimational changes in the structure of the photosynthetic apparatus via a feedback control of photosynthesis gene expression. The great variety of these signalling mechanisms is summarised under the term 'redox control'. In some cases, oxygen acts as a different environmental, light-independent stimulus of photosynthetic gene expression, providing an additional redox signal and a different kind of redox control. In this review, we summarise present knowledge about such redox control mechanisms and analyse common properties as well as differences in the various signalling pathways. We suggest that there is an urgent need for a clear distinction between different kinds of redox control. Accordingly, we propose a categorisation into perceptional and transductional redox control. These categories are defined and examples given. The generalisation and comparability of results obtained in different physiological test systems and species are critically discussed.

\section{Introduction}

Control of the expression of genes for products involved in photosynthesis is of fundamental importance for all organisms whether or not they are themselves photosynthetic. Regulatory signals influence the development and establishment of the photosynthetic machinery, its maintenance and acclimation to varying environmental conditions and its degradation during senescence. This review focuses on mechanisms that couple the reduction/oxidation (redox) state of single extra- or intracellular components to photosynthesis gene regulation. Such redox control of photosynthesis gene expression is an emerging field of research, as it appears to be a new, additional basis for light regulation in contrast to the 'classical' light-receptor-mediated gene expression control. The molecular mechanisms of primary redox-sensing steps are quite diverse and in some cases still not understood, but it has been shown that the redox state of various components influences almost all levels of photosynthetic gene expression, i.e. transcription, post-transcriptional processes and translation. Here we describe general principles of such redox control mechanisms in different photosynthetic organisms with emphasis on steps in cell signalling and the evolutionary development of photosynthetic cells. This viewpoint combines present knowledge with a new way in which to categorise and distinguish between two types of redox control.

\section{Redox control - a definition}

'Redox' reactions are chemical reactions that involve transfer of electrons or hydrogen atoms between molecules. 'Reduction' refers to the gain of one or more electrons or hydrogen atoms by an electron acceptor; 'oxidation' refers

Abbreviations - DBMIB, 2,5-dibromo-3-methyl-6-isopropyl-p-benzoquinone; LHCII, light-harvesting complex of photosystem II; PQ, plastoquinone; PS, photosynthesis; PSI, photosystem I; PSII, photosystem II; $\mathrm{Q}_{\mathrm{B}}$, quencher B; $\mathrm{Q}_{\mathrm{O}}$, quinone outer. 
to the loss of one or more electrons or hydrogen atoms by an electron donor. 'Redox state' means the oxidised or reduced state of the molecule concerned. 'Redox control' of any biological phenomenon therefore can be described as the dependency of a molecular response on the redox state of one or more of its constituent molecules. Many metabolic processes in a cell perform redox reactions and therefore a comparably large number of biological responses have been reported to be 'redox-controlled'. Long-known examples related to photosynthesis are the regulation of Calvin cycle enzymes by the thioredoxin system (reviewed by Scheibe 1991) or the mechanisms involved in scavenging oxygen radicals under light stress, such as the ascorbate-glutathione cycle (reviewed by Noctor and Foyer 1998). It has now become clear that redox control also extends to the level of gene expression. The modes of action of respective redox control initiator molecules are quite diverse and include reduction/oxidation of thiol groups, iron-sulphur centres, haems and flavins (reviewed by Bauer et al. 1999). Most of these modes of action can be found in photosynthesis gene expression. This diversity in regulated events and controlling molecular mechanisms appears to render the term 'redox control' ambiguous in describing the type of control. A simple way, however, to classify different types of redox control is to look for the position of the first controlling redox parameter within the signal transduction chain between an environmental stimulus and the respective molecular response. In a living cell this includes the perception of the environmental stimulus either through one or several receptors, a signal transduction process via an appropriate chain of transducing molecules and, ultimately, a molecular response that enables the cell to acclimate to the change in its environment (Fig. 1). We therefore may distinguish between two categories of redox control, according to the position of the controlling parameter within the signal transduction chain. Perceptional redox control occurs if the environmental stimulus (e.g. light or oxygen) itself induces a redox signal within the perceiving sensory system. Transductional redox control occurs if the perception of the stimulus (e.g. light) results in a change of the redox state of a downstream molecule of the sensory system. In both cases, the photosynthetic apparatus of photoautotrophic organisms can serve as the sensory system and, therefore, functions as a photoreceptor. In this review, we show that these types of redox control can be found in the regulation of photosynthetic gene expression. In addition, this definition appears to be valid for other redox-controlled mechanisms not covered by this review, such as the regulation of nitro-

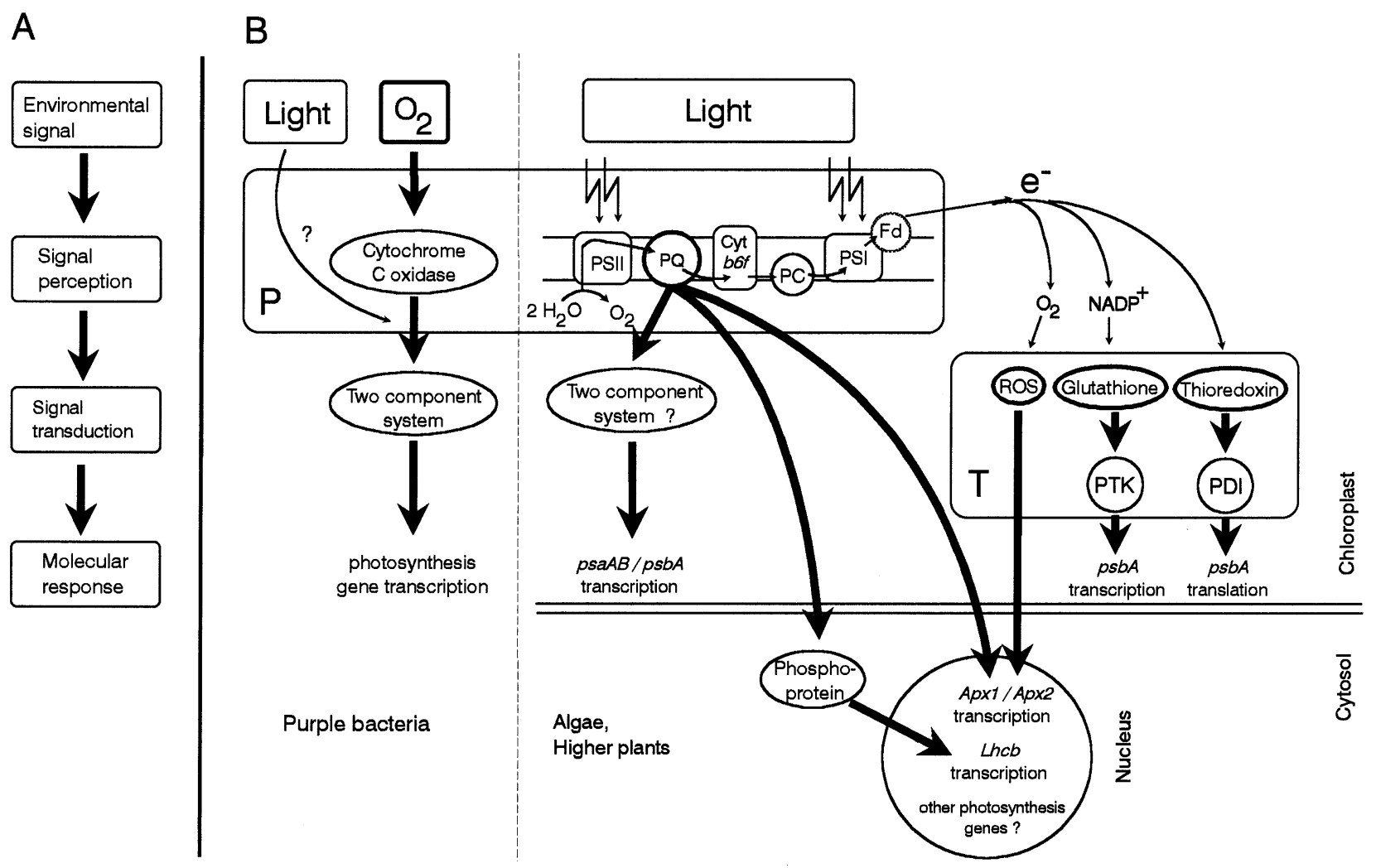

Fig. 1. Principles of redox control in PS gene expression. (A) Scheme with principal steps in cell signalling. (B) Scheme summarising the respective steps in redox control of PS gene expression. Box marked with $\mathrm{P}$ in lower left corner summarizes the sensoring systems of perceptional redox control; box marked with $\mathrm{T}$ summarizes components of transductional redox control. Only redox signalling pathways in PS gene expression events (bottom) are shown, which can be clearly attached to one of the two redox control modi (for other pathways refer to text). The respective controlling redox parameters (high-lighted by bold lines) are drawn to fit the respective step in cell signalling, i.e. signal perception or transduction. Bold black arrows represent redox-controlled signalling pathways. Uncertain or unknown connections or components are marked by a question mark. Location of gene expression events is given; the double line represents the chloroplast envelope. The oxygen-dependent redox signal occurring exclusively in purple bacteria is separated by a dotted line. 
gen fixation genes in rhizobials (reviewed by Fisher 1994) or the repression of aerobic genes under anoxia via the $\mathrm{ArcB} /$ ArcA system in E. coli (reviewed by Lynch and Lin 1996).

\section{Redox control of photosynthesis genes - a common principle in photosynthesising cells}

\section{Perceptional redox control by oxygen}

Perceptional redox control occurs if the environmental stimulus induces a redox signal within the perceiving sensory system. Among photosynthetic organisms, oxygen has been identified to be such an environmental stimulus; however, as known so far, only for purple bacteria. These facultative photoheterotrophic organisms obtain their energy either from light (via photosynthesis) or from organic and inorganic compounds depending on their environmental conditions. Extensive studies on Rhodobacter capsulatus (reviewed by Bauer and Bird 1996) and on Rhodobacter sphaeroides (reviewed by Eraso and Kaplan 2000) demonstrated that transcription of photosynthetic genes (including those for carotenoid and bacteriochlorophyll biosynthesis, for the light harvesting I [puf operon] and II [puc operon] genes and for the reaction centre genes [puh operon]) occurs under anaerobic conditions, while the presence of oxygen inhibits their expression. Oxygen appears to be the primary, light-independent signal and its action is further modified by the light intensity. Transcription of puf and puh operons is enhanced by illumination at low intensity, suggesting that endogenous redox signals from the photosynthetic apparatus may have a regulatory impact on photosynthesis gene expression. This suggests the existence of a possible perceptional or transductional redox control in purple bacteria, which, in turn, influences the perception and transduction of the primary oxygen signal. Molecular oxygen may be sensed by a $\mathrm{Cbb}_{3}$-type cytochrome $c$ oxidase and the signal is further transduced by either a two-component system (RegB-RegA in R. capsulatus, PrrB-PrrA in R. sphaeroides) to activate or, by the repressive factor Crtf, to inactivate PS gene expression. The interaction between light intensity and the oxygen-sensing system is mediated by a transcription factor called HvrA. The mode of action of these regulators is not yet fully understood. An analogous oxygen-sensing system can be found in the ArcB-ArcA two-component system of E. coli (reviewed by Lynch and Lin 1996). Further parallels are also visible in the oxygen-sensing systems of mammalian cells (reviewed by Semenza 1999).

\section{Perceptional redox control by light}

Redox regulation by the environmental factor light is very common among photosynthetic organisms and can be found in cyanobacteria, unicellular algae and higher plants. In all cases studied, changes in illumination (i.e. light quality or quantity) were used to influence electron transport in the thylakoid membrane in vivo, which, in turn, results in the change of the redox state of components of the photosynthetic machinery. In addition, temperature has been shown to be very important in modulating the incoming light signal especially at low temperatures (see below). To exclude the action of cytosolic photoreceptors and to identify the signal within the photosynthetic electron transport, site-specific electron transport inhibitors, such as 3-(3',4'dichlorophenyl)-1,1'-dimethyl urea (DCMU) and 2,5-dibromo-3-methyl-6-isopropyl-p-benzoquinone (DBMIB) are used. Responses to these inhibitors also locate the signalgenerating component within the thylakoid membrane, since DCMU blocks photosynthetic electron transport at the quencher $\mathrm{B}\left(\mathrm{Q}_{\mathrm{B}}\right)$ site of photosystem II (PSII), while DBMIB blocks at the quinone outer $\left(\mathrm{Q}_{\mathrm{O}}\right)$ site of the cytochrome $b_{6} f$ (cyt $b_{6} f$ ) complex. As DBMIB also binds to the $Q_{B}$ site if it is used in high concentrations (Trebst 1980), experiments have to be performed very carefully to avoid ambiguous or misleading results.

Studies with changing light qualities generally involve low light conditions, thus avoiding side effects of photoinhibition or other light stress functions. In chloroplast-containing organisms under such conditions, only effects on plastid genes have been reported so far. In contrast, studies that used changes in light quantity predominantly show perceptional redox effects on nuclear PS genes. In both cases, several lines of evidence identified the plastoquinone (PQ) pool and/or the cyt $b_{6} f$ complex as the most probable redox sensors (discussed below).

Changes in light quality lead to preferential absorption of light by either photosystem I (PSI) or PSII. Under conditions favouring PSII, the redox system(s) are predominantly reduced, while under conditions favouring PSI, they are predominantly oxidised. Prolonged illumination with these light qualities causes an adjustment of photosystem stoichiometry, which counteracts the imbalance in excitation energy. Extensive studies with the cyanobacterium Synechocystis PCC 6714 suggest that photosystem stoichiometry is modified by variation in the number of PSI particles. It has been shown that this adjustment is controlled by the redox state of the cyt $b_{6} f$ complex, which controls translation of the psaAB genes (reviewed by Fujita 1997). In vivo studies on Sinapis alba demonstrated that photosystem stoichiometry is adjusted by simultaneous variation of the density of both PSI and PSII in higher plants. Furthermore, in contrast to cyanobacteria, in organello studies indicated that the redox state of the PQ pool specifically controls the transcription of chloroplast-encoded photosystem core protein genes ( $p s a A B, \mathrm{P} 700$ apoproteins of PSI and $p s b A$, D1 protein of PSII), while other chloroplast genes remained unaffected (Pfannschmidt et al. 1999a,b). In its reduced state (when PSI is rate-limiting) the PQ pool activates the expression of $p s a A B$ and, in its oxidised state (when PSII is rate-limiting), the PQ pool activates the expression of $p s b A$ and represses that of $p s a A B$. It has to be noted that cyanobacteria are the only organisms that perform oxygenic photosynthesis and respiration within the same cell compartment and both electron transport chains share some common components, including the PQ pool (reviewed by Scherer 1990, Schmetterer 1994). Reduction of the PQ pool therefore occurs not exclusively by PSII, but also by stromal electron sources via NADH dehydrogenases. There is also accumulating evidence that the redox state of the PQ pool in chloroplasts of higher plants can be influenced by stromal 
components during chlororespiration. In the dark, it can be reduced by reduction equivalents via the NDH complex (Feild et al. 1998, Kofer et al. 1998). In addition, it was found that PQ can act as a electron carrier between thylakoid NADH dehydrogenase and peroxidase (Casano et al. 2000). Therefore, the role of the PQ pool in redox control of photosystem stoichiometry in cyanobacteria and chloroplasts of higher plants might be different in some details and general conclusions and comparisons must be drawn carefully.

Light quality effects on the expression of nuclear PS genes have not yet been demonstrated, but such effects do occur in response to changes in light intensity. The expression of Lhcb genes (encoding chlorophyll-binding proteins of lightharvesting complex of PSII [LHCII]) in the unicellular alga Dunaliella tertiolecta can be stimulated by an oxidised PQ pool (established by a switch from high to low light intensities). This conclusion was supported by experiments with site-specific inhibitors, such as DCMU and DBMIB (Escoubas et al. 1995). In a different approach using the related alga Dunaliella salina, it was investigated how this organism responds to changes in incident light quantity under a controlled temperature environment (Maxwell et al. 1995). This work took into account that high light intensity effects can be mimicked under low light intensities by low temperature. A decrease in temperature results in the depression of enzymatic reactions, such as the Calvin cycle, which subsequently leads to an increase in excitation pressure of PSII as it occurs under high light at moderate temperature (reviewed by Huner et al. 1998). From their results, the authors concluded that most probably the redox state of the PQ pool controls the transcription of nuclear Lhcb genes. The results from these two independent studies suggest the existence of a regulatory pathway starting from the PQ pool and leading to an increase in the amount of light-harvesting complexes and, subsequently, the quantum yield of the PS apparatus of Dunaliella species under low light or PQ oxidising conditions. First data show that a cytosolic protein becomes phosphorylated (phosphoprotein, Fig. 1) under low light conditions, which then binds to the Lhcb promoter (Escoubas et al. 1995). It therefore may be the functional link between the redox state of the PQ pool and Lhcb transcription. However, its exact role in this pathway remains to be elucidated.

In higher plants, investigations with transgenic tobacco showed that transcription of a pea ferredoxin transcribed region (Fed-1) was increased after illumination of dark-acclimated plants (Petracek et al. 1997). DCMU treatment blocked this increase as well as the polyribosome loading of this mRNA. Interestingly, light-induced accumulation of Lhcb transcripts remained unaffected in this system. Further studies using a Fed-1 construct with the tetracyclin-repressible promoter showed that disruption of photosynthetic electron transport either by darkness or by DCMU treatment leads to rapid destabilisation of the Fed-1 mRNA (Petracek et al. 1998), suggesting that this destabilisation is an immediate response to inhibition of electron transport. However, since the controlling redox component was not identified, it is not clear yet if Fed-1 gene expression is controlled by a perceptional or transductional redox signal.
Studies with Arabidopsis suggested that the reduced PQ pool generated by a low-to-high light switch partially controls the expression of nuclear-encoded Apx genes. These genes encode cytosolic ascorbate peroxidases, which are thought to be part of a high light stress defence (Karpinski et al. 1997). This high light stress response was accompanied by an increase in the ratio of oxidised to reduced glutathione, an important fact for transductional redox control mechanisms, as discussed later.

Observations of redox effects induced by light quantity changes on pure chloroplast gene expression are still rare. First, results derive from studies with Lactuca sativa after illumination of dark-acclimated plants. The authors concluded that an oxidised cyt $b_{6} f$ complex activates the overall transcriptional activity in chloroplasts (Pearson et al. 1993), but no gene-specific effects were sought. The only additional study that also used dark-light switches has been performed with Chlamydomonas chloroplasts and is focused on the translation of a single gene, i.e. $p s b A$ (Trebitsh et al. 2000), a transductional redox control, as discussed below.

In cyanobacteria, in contrast, changes in light quantity have been shown to control transcription of the $p s b A$ gene differentially. $p s b A$, unlike in higher plants and algae, is present in cyanobacteria as a gene family (reviewed by Golden 1994). Recent work suggests the involvement of the cyt $b_{6} f$ complex in the perceptional redox control of $p s b A I I /$ III transcript accumulation in Synechocystis PCC 6803 (Alfonso et al. 2000), complementing the work of Fujita and co-workers (Fujita 1997) performed with Synechocystis PCC 6714. Both strains show the same $p s b A$ gene expression characteristics, i.e. no $p s b A I$ expression at all and variable psbAII/III expression. In contrast, in Synechococcus PCC 7942, psbAI is expressed under low light and psbAII/III under high light or stress. The exchange of these transcript pools is probably dependent on the thiol redox state of the cells (Sippola and Aro 1999) and is therefore an example of transductional redox control, as defined below. Control of $p s b A$ expression is the best-known example of species-specific differences in the redox regulation of the same physiological event.

\section{Transductional redox control}

Transductional redox control occurs if the perceived stimulus leads to a change in the redox state of a downstream molecule of the sensory system. This type of redox control has been described for unicellular algae and higher plants. Studies in many laboratories focus on redox agents, which are reduced or oxidised by the electron transport chain (such as thioredoxin or glutathione) and which ultimately influence components involved in PS gene expression. Experimentally, different redox agents are applied to activity tests with the purified enzyme of interest. While these data are based on in vitro observations, their physiological importance often remains to be determined.

In the unicellular green alga Chlamydomonas reinhardtii, it was shown that binding of a translation-activating protein complex to the $p s b A$ mRNA $5^{\prime}$-untranslated region is enhanced under reducing conditions in vitro (Danon and 
Mayfield 1994), probably by means of activation by reduced thioredoxin. Further studies suggested the involvement of the disulphide isomerase-like enzyme RB60 (PDI, Fig. 1) as transducing redox active enzyme activity (Kim and Mayfield 1997). The first physiological support for this redox pathway came from a PSI-deficient mutant, which shows a reduced $p s b A$ translation. Recent in organello experiments show that light is indeed able to reduce RB60 and to enhance $p s b A$ translation. These results support the idea of light-controlled $p s b A$ translation via the ferredoxin-thioredoxin system (Trebitsh et al. 2000). In addition, electron transport efficiency also appears to influence the splicing of the $p s b A$ message in Chlamydomonas (Deshpande et al. 1997), but the present data do not distinguish between perceptional and transductional redox mechanisms. Finally, the application of DCMU or redox agents to Chlamydomonas chloroplast transformant lines MU7 grown under dark/light cycles indicates a regulatory role of photosynthetic electron transport and redox state on the degradation of chloroplast transcripts, probably via the ferredoxin-thioredoxin system (Salvador and Klein 1999).

In higher plants, it was shown that the activity of the plastid RNA processing enzyme p54 from mustard is influenced by reducing agents in vitro (Liere and Link 1997). The same is true for the plastid transcription kinase (PTK, Fig. 1) from mustard, which catalyses phosphorylation of sigmalike factors in vitro (Baginsky et al. 1997). PTK activity is enhanced in vitro by reduced glutathione (Baginsky et al. 1997, 1999). Glutathione is thought to play an important role in enhancing the expression of the $p s b A$ gene after high light stress, i.e. under increased amounts of oxidised glutathione (Karpinski et al. 1997). Enhancement of $p s b A$ gene expression should help to replenish D1 protein under high light intensities (Link 1999).

Finally, a recent in vivo study with transgenic Arabidopsis plants harbouring an Apx::Luc fusion suggests that the expression of the transgene can be influenced by reactive oxygen species (ROS), such as $\mathrm{H}_{2} \mathrm{O}_{2}$, in darkness. Thus, $\mathrm{H}_{2} \mathrm{O}_{2}$ can replace high light intensities and is claimed to act as a second messenger that allows intercellular communication, producing systemic acclimation (Foyer and Noctor 1999, Karpinski et al. 1999). In this context, it is interesting to note that the $\mathrm{H}_{2} \mathrm{O}_{2}$-generating chloroplast superoxide dismutase from mustard can be purified as a complex with the plastid RNA polymerase A (Pfannschmidt et al. 2000). The reason for such an association is not yet clear, but there is definitely a need for efficient protection of newly synthesised transcripts against ROS generated by the photosynthetic electron transport. Therefore, it is possible that the $\mathrm{H}_{2} \mathrm{O}_{2}$ produced by the action of this enzyme could act as a signal that has an impact on chloroplast transcription.

\section{Redox signalling pathways - open questions}

Present knowledge suggests that redox components initiate important signalling pathways, which ultimately regulate PS gene expression. Two questions are crucial for an understanding of this phenomenon at the molecular level.
1. How are the redox signals transduced to their respective target genes?

2. Do the various redox effects observed in different in vivo and in vitro systems reflect an integrated redox signalling network or do they represent separate signalling pathways, which operate independently from each other?

Perceptional redox signal transduction in oxygen-controlled PS gene expression of purple bacteria is quite well understood, even if details remain to be clarified. In contrast, our understanding of the signal systems under perceptional redox control starting from the PQ pool and/or the cyt $b_{6} f$ complex is poor. The large number of different organisms as well as physiological test systems makes it difficult to draw general conclusions, in particular, since species-specific mechanisms cannot be excluded at present. Nevertheless, common features and questions are obvious. First, how is the redox signal transferred out of the thylakoid membrane? It was proposed that a putative factor, whose activity depends directly on the redox state of the $\mathrm{Q}_{\mathrm{O}}$ site in cyt $b_{6}$, activates PsaA/B synthesis (reviewed by Fujita 1997). This seems to be supported by observations that show that the primary redox signal of the PQ pool is mediated via the cyt $b_{6} f$ complex (Anderson et al. 1997, Vener et al. 1997, Zito et al. 1999), but the latter investigations are focused on the redox activation of the LHCII kinase (reviewed by Allen 1992, Vener et al. 1998) and not on redox effects on gene expression. A further potential candidate for the transduction of the $\mathrm{PQ} /$ cyt $b_{6} f$ redox signal may be the thylakoid-associated kinase (Snyders and Kohorn 1999). It is still unclear whether state transitions and changes in chloroplast transcription are controlled by two different pathways or by two different branches initiated by the same redox sensor (Nilsson and Allen 1997). However, in mustard, these two processes are responses to changes in the PQ redox state, which occur on the same time scale (Allen and Pfannschmidt 2000), i.e. both responses can be observed within minutes. This suggests that they act functionally in parallel with the state transition as the gate triggering the gene expression response.

It was also hypothesised that the redox state of the PQ is sensed by a membrane-bound, two-component sensor kinase, which transfers the signal to a response regulator, which, in turn, affects gene expression (Allen 1993a,b). In cyanobacteria and purple bacteria, two-component systems are known to be involved in PS gene expression (Allen 1993c). Recent evidence strongly implicates a redox twocomponent system in both state transitions and photosystem stoichiometry adjustment in the cyanobacterium Synechocystis 6803 ( $\mathrm{Li}$ and Sherman 2000). Histidine sensor kinases have also been identified in the cytoplasm of higher plants, such as ethylene- and cytokinine-sensing kinases, and a still growing number of nuclear-encoded response-regulator-like genes have been identified in Arabidopsis and maize (for review see Chang and Stewart 1998, D'Agostino and Kieber 1999, Sakakibara et al. 2000, Urao et al. 2000). However, none of the identified genes (Imamura et al. 1999) show typical chloroplast transit peptides with the ChloroP program (Emanuelsson et al. 1999, Pfannschmidt and Oelmüller, unpublished results). The functional and molecular 
analysis of plant response-regulator-like proteins is just at its beginning, and further studies will clarify their putative role in cellular redox signalling mechanisms.

In contrast, transductional control of the chloroplast gene $p s b A$ via the ferredoxin-thioredoxin system and the translation initiation complex in Chlamydomonas (Danon and Mayfield 1994, Kim and Mayfield 1997) or via glutathione and the RNA polymerase kinase complex in mustard (Baginsky et al. 1997) appears to be better understood. Components for the transduction of the redox signal to the gene expression level are known and appropriate models of their action are proposed (reviewed by Link 1999). Further studies will show if these models apply in vivo and if other plastid genes besides $p s b A$ are regulated in the same way.

Redox control of the expression of nuclear PS genes represents a second major task in plant research, as the signal has to pass through the chloroplast envelope. The mechanisms by which the signal is transported over the membrane are completely unknown to date. In addition, it is not clear if there exist different redox signals and, if yes, how many. The various physiological test systems (e.g. light quantity or light quality), which can be used to investigate redox signals, suggest the existence of at least several signals. In any case, redox signals may represent one form of the so-called 'plastid factor' (Oelmüller 1989), whose nature is still unknown (reviewed by Taylor 1989, Goldschmidt-Clermont 1998). As already mentioned, studies with Dunaliella tertiolecta point to the involvement of a phosphorylatable protein messenger (Escoubas et al. 1995, Durnford and Falkowski 1997) in this interorganellar communication. Recent studies with the cue1 (cab underexpressed) mutant from Arabidopsis suggest also the involvement of a plastid phosphoenolpyruvate/phosphate translocator in this pathway (Streatfield et al. 1999).

As chloroplast metabolism and photosynthesis are tightly coupled, it is possible that both contribute to the chloroplast signal. An important difference in redox control of nuclear and chloroplast PS genes, at least in higher plants, is that the redox signal passing to the nucleus is composed of about $80-120$ individual signals (depending on the total number of chloroplasts in the respective cell), while the redox control within each chloroplast is organelle-specific. As the redox poise of the chloroplasts within one cell may differ because of their cellular position, nuclear gene expression reflects the response to an average of all these signals. Therefore, chloroplast redox signals to the nucleus contain more general information about the photosynthetic capacity of the cell, while redox signals within the plastid allow control of gene expression according to the specific situation within a single chloroplast. Answers to these questions could be obtained by comparison of redox pathways in cells with multiple chloroplasts (e.g. higher plants) and in cells with a single chloroplast, such as Chlamydomonas or the fts $Z \mathrm{mu}-$ tant of Arabidopsis (Osteryoung et al. 1998).

As outlined above, redox control in chloroplasts starts from different redox parameters. How do these various redox signals contribute to the gene-specific control of PS gene expression in a coordinated way? We still need more information to understand this mechanism. Nevertheless, it appears that a hierarchical order of the various redox sig-
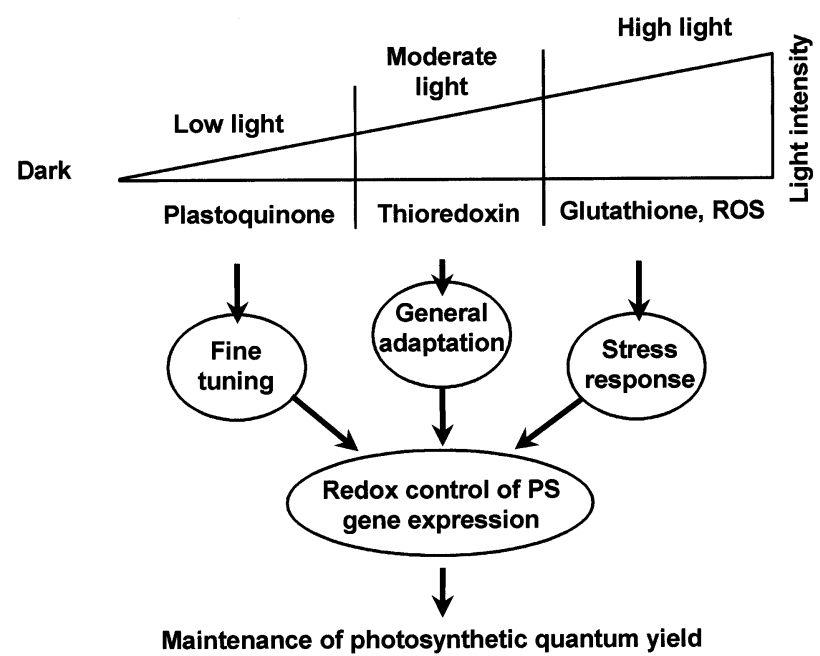

Fig. 2. Simplified model of hierarchical order in light-dependent redox control of PS gene expression in a photosynthetic cell. The triangle represents an increase in incident light intensity from the left to the right. The light intensity and the respective main active redox control parameter are correlated by perpendicular lines representing 3 light intensity windows. Each parameter has to fulfil a different physiological role according to the light intensity, which in the end helps to acclimate photosynthesis to changing illumination.

nals influences gene expression according to the incident light intensity (Fig. 2). Our general assumption is that a redox control parameter is active only under those light intensities under which it shows variable ratios in its oxidised versus reduced forms. A model is based on 3 light intensity windows, i.e. low, moderate and high light intensity. The range of the low light intensity is defined by the redox state of the PQ pool, which depends directly on the linear electron transport. Its main physiological role is to redistribute imbalances in energy excitation between the two photosystems to provide an efficient electron flux, even under limited photon yields, such as under water, in shade or under cloud, by means of activation of physiological variations or gene expression mechanisms.

Reaching the point of complete PQ reduction by increasing light intensity, the second window becomes activated, in which we suggest that thioredoxin may become the predominant redox control parameter. Thioredoxin is reduced by ferredoxin via a ferredoxin-thioredoxin reductase, a crucial step in photosynthesis redox chemistry, since the received electron signal from ferredoxin is transduced into a thiol signal transmitted to thioredoxin (Dai et al. 2000; reviewed by Schürmann and Jacquot 2000). The redox state of thioredoxin may still be variable under moderate light intensities, as it depends on the linear and cyclic electron transport. In addition, the midpoint redox potential $(\mathrm{Em})$ of thioredoxin $(-290 \mathrm{mV}$ for thioredoxin $\mathrm{f}$ and $-300 \mathrm{mV}$ for thioredoxin $\mathrm{m}$, Schürmann and Jacquot 2000) is more negative than that of PQ $(\sim+100 \mathrm{mV})$. According to the Nernst equation, under a constant $\mathrm{pH}$ and equal total concentration (of PQ and thioredoxin), PQ has a ratio of reduced to oxidised forms of $10^{7}$ when at equilibrium with thioredoxin that is exactly $50 \%$ reduced. Differences in the redox potential of the enzymes of the Calvin cycle suggest a sequential activa- 
tion of these enzymes by the reduction state of thioredoxin as well as different degrees of activation (Faske et al. 1995). Therefore, thioredoxin may act in a similar way as regulator of gene expression.

The third proposed window describes conditions in which the incident light intensity increases such that thioredoxin remains reduced. As a consequence, dissipation of excess light energy becomes important in order to avoid photooxidative damage. High light stress therefore increases the GSSG/GSH ratio as well as ROS production, such as $\mathrm{H}_{2} \mathrm{O}_{2}$ (reviewed by Noctor and Foyer 1998). Increasing concentrations of GSSG or $\mathrm{H}_{2} \mathrm{O}_{2}$ then act as the activation signals for light stress defence mechanisms. The width of each light intensity window depends on the photosynthetic capacity of the individual organism, which explains why a given gene under the same conditions is controlled by different redox mechanisms in different species. It is also important to note that the action of the 3 proposed redox mechanisms may overlap to a certain degree.

\section{Evolution of photosynthetic cells and its impact on redox signals}

As described above, photosynthetically active cells from bacteria to higher plants contain various redox-controlled mechanisms to regulate the expression of photosynthesis genes. Purple and cyanobacteria show immediate responses to environmental changes and these changes are necessary for their survival. Since these organisms are related to the chloroplast of eukaryotic plant cells, it will not be surprising that these mechanisms are still active in higher plants. Since chloroplasts contain rather little genetic information (the plastome encodes approximately 80 polypeptides, while more than 2500 nuclear genes appear to code for additional plastid proteins (Abdallah et al. 2000), diversification of redox signals within the eukaryotic cell may be expected to have occurred (Fig. 3). The total on-off redox mechanism in purple bacteria was dispersed in various signals starting from the chloroplast electron transport chain to control the genetic information for the photosynthetic apparatus when it became divided between nucleus and plastome. For a higher plant cell, the sum of all redox signals starting from the about $80-120$ chloroplasts may be the most important signal for the regulated expression of the nuclear-encoded photosynthetic genes. The chloroplast itself still has control over the biogenesis of photosynthetic core proteins, which may be the pacemaker for the synthesis and assembly of photosynthetic centres. This fact might be the reason for retaining a chloroplast genome during evolution (Allen 1993a,b, Pfannschmidt et al. 1999a, Race et al. 1999). Interestingly, ROS seem to have a regulatory role in tissueoverriding redox signalling, i.e. the ROS signal of one cell is an exogenous redox signal for another cell. This is reminiscent of the oxygen response of purple bacteria, suggesting that this signalling pathway may have been converted in an intercellular pathway during evolution.

In this way, photosynthesis itself can be understood as a receptor for environmental information controlling the expression of the nuclear and plastid-encoded genes encoding the components that are necessary for an efficient photosynthetic process. The concept of photosynthesis as sensor for environmental information has been originally introduced as the 'grand design of photosynthesis' by Arnon (1982) and further extended by Anderson et al. (1995) and Huner et al. (1998). The present data about redox control of photosynthesis gene expression are consistent with this concept and indicate its important role as regulator of plant metabolism and gene expression.

\section{Future perspectives}

Our knowledge about light-dependent redox signal mechanisms controlling gene expression is growing. The present focus still lies on genes encoding proteins involved in or related to photosynthetic functions, but it is very likely that other light-regulated genes may also be controlled by redox mechanisms, such as nitrate reductase or nitrogenase. Furthermore, it must be considered that illumination oscillates in a daily rhythm and, therefore, it can be expected that the action of redox control mechanisms is also influenced in such a way. A role for redox signals in the circadian control of gene expression may be anticipated, at least in defining the amplitude of the oscillation.

Besides the chloroplast, mitochondria perform many of the redox reactions in the cell. The electron flow through the mitochondrial respiratory chain is light-independent and may contribute to completely different genes and redox signalling mechanisms. Studies in potato mitochondria show that the redox state of the respiratory chain has an impact on mitochondrial RNA synthesis (Wilson et al. 1996). In addition, it has been shown that mitochondrial respiration also contributes to photosynthetic capacity in winter rye by using reducing equivalents from linear photosynthetic elec-
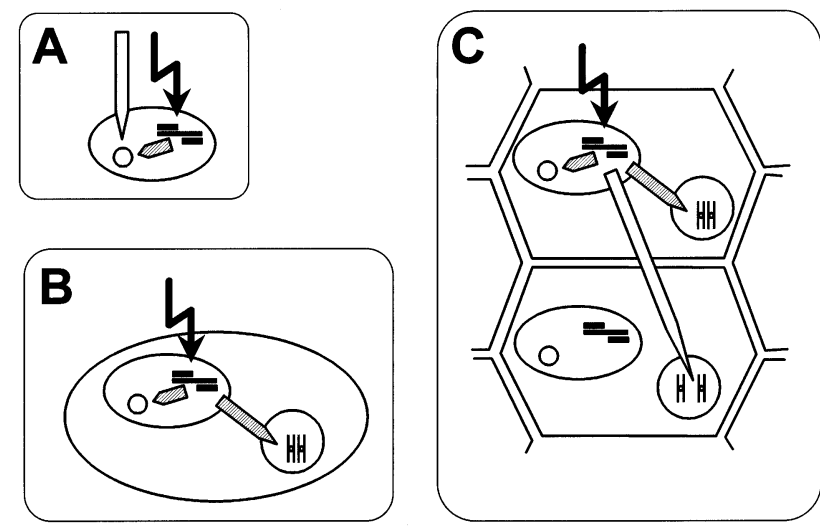

Fig. 3. Diversification of redox signals within photosynthetic cells during evolution. Redox control pathways of PS gene expression in photosynthetic bacteria representing the ancestors of chloroplasts (A), in unicellular algae (B) and in plant tissue (C). The evolutionary organisation of the respective photosynthetic organism is indicated by the shape and size of the cell. Open arrows represent exogenous redox signals from the environment of the cell, hatched arrows are redox signals within the cell and black zig-zag arrows represent light. The photosynthetic machinery is represented by 3 stacked black lines, bacterial or plastid DNA by a small circle and the nuclear compartment by a bigger circle containing two chromosomes, respectively. 
tron transport (Hurry et al. 1995). Furthermore, it has been shown in pea protoplasts that respiration helps to protect photosynthesis from photoinhibition (Saradadevi and Raghavendra 1992). So there are clear hints that there exists an interdependence between photosynthesis and mitochondrial respiration not only in cyanobacteria (see above), but also in plant cells, where both events take place in different compartments (reviewed by Raghavendra et al. 1994). The future will show how these signals contribute to the redox signalling network of photosynthetic cells and to interorganellar communication.

Acknowledgements - We thank G. Link for providing a preprint manuscript prior publication. Work in the laboratories of T. P. and R. O. in Jena is supported by the Deutsche Forschungsgemeinschaft and the Fond der Chemischen Industrie. J. F. A. thanks the Swedish Natural Sciences Research Council (NFR) and the Crafoord Foundation for support.

\section{References}

Abdallah F, Salamini F, Leister D (2000) A prediction of the size and evolutionary origin of the proteome of chloroplasts of Arabidopsis. Trends Plant Sci 5: 141-142

Alfonso M, Perewoska I, Kirilovsky D (2000) Redox control of $p s b A$ gene expression in the cyanobacterium Synechocystis PCC 6803. Involvement of the cytochrome $b_{6} / f$ complex. Plant Physiol 122: $505-515$

Allen JF (1992) Protein phosphorylation in regulation of photosynthesis. Biochim Biophys Acta 1098: 275-335

Allen JF (1993a) Control of gene expression by redox potential and the requirements for chloroplast and mitochondrial genomes. J Theor Biol 165: 609-631

Allen JF (1993b) Redox control of gene expression and the function of chloroplast genomes - an hypothesis. Photosynth Res 36: 95-102

Allen JF (1993c) Redox control of transcription: Sensors, response regulators, activators and repressors. FEBS Lett 332: 203-207

Allen JF, Pfannschmidt T (2000) Balancing the two photosystems: Photosynthetic electron transfer governs transcription of reaction centre genes in chloroplasts. Phil Trans R Soc B 1402 $1351-1359$

Anderson JM, Chow WS, Park Y-I (1995) The grand design of photosynthesis: Acclimation of the photosynthetic apparatus to environmental cues. Photosynth Res 46: 129-139

Anderson JM, Price GD, Chow WS, Hope AB, Badger MR (1997) Reduced levels of cytochrome $b f$ complex in transgenic tobacco leads to marked photochemical reduction of the plastoqinone pool, without significant change in acclimation to irradiance. Photosynth Res 53: 215-227

Arnon DI (1982) Sunlight, earth, life: The grand design of photosynthesis. Sciences 22: $22-27$

Baginsky S, Tiller K, Link G (1997) Transcription factor phosphorylation by a protein kinase associated with chloroplast RNA polymerase from mustard (Sinapis alba). Plant Mol Biol 34: $181-189$

Baginsky S, Tiller K, Pfannschmidt T, Link G (1999) PTK, the chloroplast RNA polymerase associated protein kinase from mustard (Sinapis alba), mediates redox control of plastid in vitro transcription. Plant Mol Biol 39: 1013-1023

Bauer CE, Bird TH (1996) Regulatory circuits controlling photosynthesis gene expression. Cell 85: 5-8

Bauer CE, Elsen S, Bird TH (1999) Mechanisms for redox control of gene expression. Annu Rev Microbiol 53: 495-523

Casano LM, Zapata JM, Martin M, Sabater B (2000) Chlororespiration and poising of cyclic electron transport. Plastoquinone as electron transporter between thylakoid NADH dehydrogenase and peroxidase. J Biol Chem 275: 942-948

Chang C, Stewart RC (1998) The two-component system. Regulation of diverse signalling pathways in prokaryotes and eukaryotes. Plant Physiol 117: 723-731
D'Agostino IB, Kieber J (1999) Phosphorelay signal transduction: The emerging family of plant response regulators. Trends Biol Sci 24: $452-456$

Dai S, Schwendtmayer C, Schürmann P, Ramaswamy S, Eklund H (2000) Redox signalling in chloroplasts: Cleavage of disulfides by an iron-sulfur cluster. Science 287: 655-658

Danon A, Mayfield SP (1994) Light-regulated translation of chloroplast messenger RNAs through redox potential. Science 266: $1717-1719$

Deshpande NN, Bao Y, Herrin DL (1997) Evidence for light/redoxregulated splicing of $p s b A$ pre-RNAs in Chlamydomonas chloroplasts. RNA 3: $37-48$

Durnford DG, Falkowski PG (1997) Chloroplast redox regulation of nuclear gene transcription during photoacclimation. Photosynth Res 53: 229-241

Emanuelsson O, Nielsen H, von Heijne G (1999) ChloroP, a neural network-based method for predicting chloroplast transit peptides and their cleavage sites. Protein Sci 8: 978-984

Eraso JM, Kaplan S (2000) From redox flow to gene regulation: Role of the PrrC protein of Rhodobacter sphaeroides 2.4.1. Biochemistry 39: 2052-2062

Escoubas J-M, Lomas M, LaRoche J, Falkowski PG (1995) Light intensity regulation of $c a b$ gene transcription is signalled by the redox state of the plastoquinone pool. Proc Natl Acad Sci USA 92: $10237-10241$

Faske M, Holtgrefe S, Ocheretina O, Meister M, Backhausen JE, Scheibe R (1995) Redox equilibria between the regulatory thiols of light/dark-modulated chloroplast enzymes and dithiothreitol: Fine-tuning by metabolites. Biochim Biophys Acta 1247: 135142

Feild TS, Nedbal L, Ort DR (1998) Nonphotochemical reduction of the plastoquinone pool in sunflower leaves originates from chlororespiration. Plant Physiol 116: 1209-1218

Fisher H-M (1994) Genetic regulation of nitrogen fixation in Rhizobia. Microbiol Rev 58: 352-386

Foyer CH, Noctor G (1999) Plant biology - leaves in the dark see light. Science 284: 599-601

Fujita Y (1997) A study on the dynamic features of photosystem stoichiometry: Accomplishments and problems for future studies. Photosynth Res 53: 83-93

Golden SS (1994) Light-responsive gene expression and the biochemistry of the photosystem II reaction centre. In: Bryant DA (ed) The Molecular Biology of Cyanobacteria. Kluwer Academic Publishers, Dordrecht, pp 693-714

Goldschmidt-Clermont M (1998) Coordination of nuclear and chloroplast gene expression in plant cells. Int Rev Cytol 117: $115-180$

Huner NPA, Öquist G, Sarhan F (1998) Energy balance and acclimation to light and cold. Trends Plant Sci 3: 224-230

Hurry V, Tobiaeson M, Krömer S, Gardeström P, Öquist G (1995) Mitochondria contribute to increased photosynthetic capacity of leaves of winter rye (Secale cereale L.) following cold-hardening. Plant Cell Environ 18: 69-76

Imamura A, Hanaki N, Nakamura A, Suzuki T, Taniguchi M, Kiba T, Ueguchi C, Sugiyama T, Mizuno T (1999) Compilation and characterisation of Arabidopsis thaliana response regulators implicated in His-Asp phosphorelay signal transduction. Plant Cell Physiol 40: 733-742

Karpinski S, Escobar C, Karpinska B, Creissen G, Mullineaux PM (1997) Photosynthetic electron transport regulates the expression of cytosolic ascorbate peroxidase genes in Arabidopsis during excess light stress. Plant Cell 9: 627-640

Karpinski S, Reynolds H, Karpinska B, Wingsle G, Creissen G, Mullineaux PM (1999) Systemic signalling and acclimation in response to excess excitation energy in Arabidopsis. Science 284: 654-657

Kim J, Mayfield SP (1997) Protein disulfide isomerase as a regulator of chloroplast translational activation. Science 278: 1954-1957

Kofer W, Koop HU, Wanner G, Steinmuller K (1998) Mutagenesis of the genes encoding subunits $\mathrm{A}, \mathrm{C}, \mathrm{H}, \mathrm{I}, \mathrm{J}$ and $\mathrm{K}$ of the plastid $\mathrm{NAD}(\mathrm{P}) \mathrm{H}$-plastoquinone-oxidoreductase in tobacco by polyethylene glycol-mediated plastome transformation. Mol Gen Genet 258: 166-173

Li H, Sherman LA (2000) A redox-responsive regulator of photosynthesis gene expression in the cyanobacterium Synechocystis sp. strain PCC 6803. J Bacteriol 182: 4268-4277 
Liere K, Link G (1997) Chloroplast endoribonuclease p54 involved in RNA 3'-end processing is regulated by phosphorylation and redox state. Nucleic Acids Res 25: 2403-2408

Link G (1999) Redox regulation of photosynthetic genes. In Govindjee (ed) Advances in Photosynthesis. Kluwer Academic Publishers, Dordrecht, pp 1-52.

Lynch AS, Lin ECC (1996) Responses to molecular oxygen. In Neidhardt FC (ed) Escherichia coli and Salmonella: Cellular and Molecular Biology. ASM Press, Washington DC, pp 1526-1538

Maxwell DP, Laudenbach DE, Huner NPA (1995) Redox regulation of light-harvesting complex II and $c a b$ mRNA abundance in Dunaliella salina. Plant Physiol 109: 787-795

Nilsson A, Allen JF (1997) Redox signalling and the structural basis of regulation of photosynthesis by protein phosphorylation. Physiol Plant 100: 863-868

Noctor G, Foyer C (1998) Ascorbate and glutathione: Keeping active oxygen under control. Annu Rev Plant Physiol Plant Biol 49: $249-279$

Oelmüller R (1989) Photooxidative destruction of chloroplasts and its effect on nuclear gene expression and extraplastidic enzyme levels. Photochem Photobiol 49: 229-239

Osteryoung KW, Stokes KD, Rutherford SM, Percival AL, Lee WY (1998) Chloroplast division in higher plants require members of two functionally divergent gene families with homology to bacterial $\mathrm{fts} Z$. Plant Cell 10: 1991-2004

Pearson CK, Wilson SB, Schaffer R, Ross AW (1993) NAD turnover and utilisation of metabolites for RNA synthesis in a reaction sensing the redox state of the cytochrome $b_{6} f$ complex in isolated chloroplasts. Eur J Biochem 218: 397-404

Petracek ME, Dickey LF, Huber SC, Thompsen WF (1997) Lightregulated changes in abundance and polyribosome association of ferredoxin mRNA are dependent on photosynthesis. Plant Cell 9: 2291-2300

Petracek ME, Dickey LF, Nguyen TT, Gatz C, Sowinski DA, Allen GC, Thompsen WF (1998) Ferredoxin-1 mRNA is destabilised by changes in photosynthetic electron transport. Proc Natl Acad Sci USA 95: 9009-9013

Pfannschmidt T, Nilsson A, Allen JF (1999a) Photosynthetic control of chloroplast gene expression. Nature 397: 625-628

Pfannschmidt T, Nilsson A, Tullberg A, Link G, Allen JF (1999b) Direct transcriptional control of the chloroplast genes $p s b A$ and psaAB adjusts photosynthesis to light energy distribution in plants. Life 48: 1-6

Pfannschmidt T, Orgzewalla K, Baginsky S, Siekmann A, Meyer HE, Link G (2000) The multi-subunit chloroplast RNA polymerase A from mustard (Sinapis alba L.): Integration of a prokaryotic core into a larger complex with organelle-specific functions. Eur J Biochem 267: 253-261

Race HL, Herrmann RG, Martin W (1999) Why have organelles retained genomes? Trends Genet 15: 364-370

Raghavendra AS, Padmasree K, Saradadevi K (1994) Interdependence of photosynthesis and respiration in plant cells: Interaction between chloroplasts and mitochondria. Plant Sci 97: 1-14

Sakakibara H, Taniguchi M, Sugiyama T (2000) His-Asp phosphorelay signalling: A communication avenue between plants and their environment. Plant Mol Biol 42: 273-278
Salvador ML, Klein U (1999) The redox state regulates RNA degradation in the chloroplast of Chlamydomonas reinhardtii. Plant Physiol 121: 1367-1374

Saradadevi K, Raghavendra AS (1992) Dark respiration protects photosynthesis against photoinhibition in mesophyll protoplasts of pea (Pisum sativum). Plant Physiol 99: 1232-1237

Scheibe R (1991) Redox-modulation of chloroplast enzymes. A common principle for individual control. Plant Physiol 96: 1-3

Scherer S (1990) Do photosynthesis and respiratory electron transport chains share redox proteins? Trends Biochem Sci 15: $458-$ 462

Schmetterer G (1994) Cyanobacterial respiration. In: Bryant DA (ed) The Molecular Biology of Cyanobacteria. Kluwer Academic Publishers, Dordrecht, pp 409-435

Schürmann P, Jacquot J-P (2000) Plant thioredoxin systems revisited. Annu Rev Plant Physiol Plant Mol Biol 51: 371-400

Semenza GL (1999) Perspectives in oxygen sensing. Cell 98: $281-$ 284

Sippola K, Aro E-M (1999) Thiol redox state regulates expression of psbA genes in Synechococcus sp. PCC 7942. Plant Mol Biol 41: $425-433$

Snyders S, Kohorn BD (1999) TAKs, thylakoid membrane protein kinases associated with energy transduction. J Biol Chem 274: 9137-9140

Streatfield SJ, Weber A, Kinsman EA, Husler RE, Li J, Post-Beittenmiller D, Kaiser WM, Pyke KA, Flügge U-I, Chory J (1999) The phosphoenolpyruvate/phosphate translocator is required for phenolic metabolism, palisade cell development, and plastiddependent nuclear gene expression. Plant Cell 11: 1609-1621

Taylor WC (1989) Regulatory interactions between nuclear and plastid genomes. Annu Rev Plant Physiol Plant Mol Biol 46: $445-474$

Trebitsh T, Levitan A, Sofer A, Danon A (2000) Translation of chloroplast $p s b A$ mRNA is modulated in the light by counteracting oxidising and reducing activities. Mol Cell Biol 20: $1116-$ 1123

Trebst A (1980) Inhibitors in electron flow: Tools for the functional and structural localisation of carriers and energy conservation sites. Methods Enzymol 69: 675-715

Urao T, Yamaguchi-Shinozaki K, Shinozaki K (2000) Two-component systems in plant signal transduction. Trends Plant Sci 5: $67-74$

Vener AV, van Kann PJM, Rich PR, Ohad I, Andersson B (1997) Plastoquinol at the quinol site of reduced cytochrome $b f$ mediates signal transduction between light and protein phosphorylation: Thylakoid protein kinase deactivation by a single-turnover flash. Proc Natl Acad Sci USA 94: 1585-1590

Vener AV, Ohad I, Andersson B (1998) Protein phosphorylation and redox sensing in chloroplast thylakoids. Curr Opin Plant Biol 1: 217-223

Wilson SB, Davidson GS, Thomson LM, Pearson CK (1996) Redox control of RNA synthesis in potato mitochondria. Eur J Biochem 242: $81-85$

Zito F, Finazzi G, Delosme R, Nitschke W, Picot D, Wollman F-A (1999) The Qo site of cytochrome $b_{6} f$ complexes controls the activation of the LHCII kinase. EMBO J 18: 2961-2969 